\title{
Levodopa-carbidopa intestinal gel therapy in Parkinson's disease: Procedure complications
}

\author{
Luminita Celia Popa' ${ }^{1}$ Daniel-Corneliu Leucuta ${ }^{2}$, Nicoleta Tohanean', \\ Stefan-Lucian Popa ${ }^{3}$, Lacramioara Perju-Dumbrava ${ }^{1}$ \\ ${ }^{1}$ Neurology Department, "Iuliu Hatieganu“ University of Medicine and Pharmacy, \\ Cluj-Napoca, Romania \\ ${ }^{2}$ Department of Medical Informatics and Biostatistics, \\ "Iuliu Hatieganu" University of Medicine and Pharmacy, Cluj-Napoca, Romania \\ ${ }^{3} 2^{\text {nd }}$ Medical Department, "Iuliu Hatieganu" University of Medicine and Pharmacy \\ Cluj-Napoca, Romania
}

\begin{abstract}
Objectives. Continuous delivery of levodopa-carbidopa intestinal gel (LCIG) represents a gold standard option for the treatment of advanced PD and the benefits of intestinal gel delivery are significative superior from those obtained with available medical therapies. We aimed to investigate the percutaneous endoscopic transgastric jejunostomy (PEG-J) use and the occurrence of procedural complications in a group of patients diagnosed with PD Hoehn and Jahr 3 and 4 stages, receiving LCIG therapy.

Materials and methods. We performed an observational retrospective cohort study on 24 PD patients recruited from a single regional tertiary center in Cluj-Napoca, Romania, between 2009 and 2019. We included PD patients with Hoehn and Jahr 3 and 4 stages under LCIG therapy.

Results. PEG-J replacement was found in $54.2 \%$ of the patients. Erythema and inflammation at the level of stoma $(37.5 \%)$ and allergic reactions $(20.8 \%)$ were the most common complications. The most frequent reason for PEG-J replacement was tube dislocation (20.8\%), and rarely the plied tube, the excess of adipose tissue, or bezoar (4.3\% each). A third of the patients received PEG-J therapy during the night, after a median time of three years, after PEG-J initiation. A quarter of all the subjects had their Levodopa-Carbidopa therapy interrupted. The median time untill interruption was one year. The reasons for the interruption were patient, or patient's relative refuses, for a sixth of the cases, or gastric polyps, in very few cases.

Conclusions. The most frequent complication of LCIG delivered by PEG-J was erythema and inflammation at the level of stoma. However LCIG therapy discontinuation was in particular due to the decision of the patient or relatives.
\end{abstract}

Keywords: Parkinson's disease, PD, gastroenterological complications, levodopa-carbidopa intestinal gel (LCIG)

\section{INTRODUCTION}

Parkinson's disease (PD) is a chronic and progressive movement disorder and the second-most common neurodegenerative disorder in the world [1-4]. In 2015, 6.2 million people were diagnosed with PD, with 117,400 deaths/year globally [3].

PD is associated with progressive disability and mobility-related impairments with increased mortality and decreased quality of life for patients and their families [5-10]. If the onset of the disorder ap- pears in the fifth decade, it is called early-onset PD, but the disease typically occurs in people in the seventh decade.

The symptomatology is dominated by the motor fluctuations and the alteration between „on” (mobility often with dyskinesia) and „off” (immobility) episodes [4].

Levodopa and carbidopa represent the primary therapy of PD. Because their absorption is made in the small bowel, a decreased level of absorption 
leads to a low quantity of dopamine in striatal level, with the primary clinical manifestation represented by an „off” episode. The narrowing of the therapeutic window, after a period of efficient oral therapy, is a significant source of severe complications, including memory loss, hallucinations, delusions, sleep disturbances, anxiety, constipation or diarrhea, fecal incontinence, dysphagia, and dyskinesia.

The most crucial goal of PD therapy is to reduce "off" episodes without inducing the development of dyskinesia. Levodopa-carbidopa intestinal gel (LCIG) continuous administration through an intrajejunal percutaneous gastrostomy tube, has the effect of a more consistent dopaminergic stimulation in the brain and noticeable clinical improvement [4-10]. Continuous delivery of LCIG represents a gold standard option for the treatment of advanced PD, and the benefits of intestinal gel delivery are significantly superior from those obtained with available medical therapies.

Nevertheless, there are gastrointestinal complications associated with this method of drug administration.

This study aimed to investigate the PEG-J use and the occurrence of procedural complications in a group of patients diagnosed with PD Hoehn and Jahr 3 and 4 stages, receiving LCIG therapy.

\section{MATERIALS AND METHODS}

Study design and setting

We performed an observational retrospective cohort study on 24 patients diagnosed with PD from a single third level center, in Cluj-Napoca, Romania, between 2009 and 2019.

\section{Patients}

Inclusion criteria were PD patients with Hoehn and Jahr 3 and 4 stages receiving LCIG treatment. Exclusion criteria were: unclear diagnosis of PD, other parkinsonian syndromes, neurodegenerative diseases, concomitant narrow-angle glaucoma, having contraindications for the placement of a nasogastric probe or jejunal tube and oncological diseases.

\section{Variables}

The data was collected from medical files and the electronic records of the hospital. We collected demographic data (age, gender, place of residence),
PD symptoms and stadialization (disease duration, Hoehn and Yahr at baseline, UPDRS II and IIIinitially), PEG-J (percutaneous endoscopic transgastric jejunostomy) use, interruption and reasons for interruption during night, PEG-J placement refusal reason, interruption of intrajejunal Levodopa-Carbidopa therapy, PEG-J interruption motive, PEG-J complications, PEG-J replacement, and reasons for replacement. The unified Parkinson's disease rating scale (UPDRS) is commonly used to assess the clinical evolution of Parkinson's disease, both in hospitals but also in studies [5].

\section{Statistical analysis}

Categorical data were presented as counts and percentages. Continuous data were presented as means and standard deviations (for non-skewed data) or medians and quartiles (for skewed data). All statistical analyses were performed with the $\mathrm{R}$ environment for statistical computing and graphics (R Foundation for Statistical Computing, Vienna, Austria), version 3.6.1 [7].

\section{Ethics statement}

The study was performed in agreement with the Declaration of Helsinki and was approved by the "Iuliu Hatieganu" University of Medicine and Pharmacy Ethics Committee.

\section{RESULTS}

The study included 24 subjects with a mean age of 70.1 years (standard deviation of 7.7, ranging from 55 to 85 years). There were 13 males $(54.2 \%)$, the majority having urban residence $(21-87.5 \%)$.

The median PD duration was 15 years (25 and 75 percentiles: $13.8-20.3$ years), while the duration from the start of the therapy was 5 years ( 25 and 75 percentiles: 3.8-7 years). The ratio of the 3 and 4 Hoehn and Yahr stages was 1 to 10. The initial UPDRS II and III median values were 37 (25 and 75 percentiles: $33-39$ ) and 41.5 (25 and 75 percentiles: 38-45).

A third of the patients received PEG-J therapy during the night, after a median time of three years, after PEG-J initiation (see Table 1). A quarter of the subjects in this group had interrupted the therapy after a median time of one year, mainly due to patients, or patients' relative request. 
A quarter of all the subjects had their LCIG therapy interrupted. The median time till interruption was one year. The reasons for the interruptions were patient, or patient's relative refuses, for a sixth of the cases, or gastric polyps, in very few cases.

PEG-J replacement was found in half of the patients. Erythema and inflammation at the level of stoma and allergic reactions were the most common complications. The most frequent reason for PEG-J replacement was tube dislocation and rarely the plied tube, the excess of adipose tissue, or bezoar.

TABLE 1. Characteristics of levodopa-carbidopa intestinal gel therapy in PD patients

\begin{tabular}{|c|c|}
\hline Characteristic & $\frac{\text { Number }(\%)}{(n=24)}$ \\
\hline \multicolumn{2}{|l|}{ PEG-J during the night } \\
\hline PEG-J during the night & $8 / 24(33.33)$ \\
\hline $\begin{array}{l}\text { Number of years since the therapy introduction } \\
\text { during the night since the initiation of the } \\
\text { intrajejunal treatment, median (IQR) }\end{array}$ & $3(2-5)$ \\
\hline $\begin{array}{l}\text { Interruption of levodopa-carbidopa intrajejunal } \\
\text { treatment during the night }\end{array}$ & $2 / 24(8.33)$ \\
\hline $\begin{array}{l}\text { Reasons for the interruption of LCIG treatment } \\
\text { during the night }\end{array}$ & $2 / 24(8.33)$ \\
\hline \multicolumn{2}{|l|}{ PEG-J placement refusal reason } \\
\hline Patient decision & $2 / 24(8.33)$ \\
\hline Patient's relative decision & $2 / 24(8.33)$ \\
\hline \multicolumn{2}{|l|}{$\begin{array}{l}\text { Interruption of intrajejunal } \\
\text { levodopa-carbidopa therapy }\end{array}$} \\
\hline $\begin{array}{l}\text { Interruption of intrajejunal levodopa-carbidopa } \\
\text { therapy }\end{array}$ & $6 / 24(25)$ \\
\hline $\begin{array}{l}\text { Duration of intrajejunal treatment with } \\
\text { levodopa-carbidopa in patients who } \\
\text { interrupted the treatment and deceased } \\
\text { patients expressed in days, median (IQR) }\end{array}$ & $\begin{array}{c}364.5 \\
(3-1,460)\end{array}$ \\
\hline \multicolumn{2}{|l|}{ PEG-J interruption motive } \\
\hline Pacient refuse & $2 / 24(8.33)$ \\
\hline Patient's relative refuse & $2 / 24(8.33)$ \\
\hline Gastric polyp & $1 / 24(4.17)$ \\
\hline $\begin{array}{l}\text { Acute psychosis, or dementia, or active gastric } \\
\text { ulcer }\end{array}$ & $0 / 24(0)$ \\
\hline \multicolumn{2}{|l|}{ PEG-J complications } \\
\hline $\begin{array}{l}\text { Erythema and inflammation at the level of } \\
\text { stoma }\end{array}$ & $9 / 24(37.5)$ \\
\hline Local allergic reaction to disinfectant & $5 / 24(20.83)$ \\
\hline \multicolumn{2}{|l|}{ PEG-J replacement } \\
\hline PEG-J Replacement & $13 / 24(54.17)$ \\
\hline Number of replacements PEG-J, median (IQR) & $0(0-1)$ \\
\hline \multicolumn{2}{|l|}{ The reason for PEG-J replacement } \\
\hline Tube dislocation & $8 / 23(34.78)$ \\
\hline Excess of adipose tissue & $1 / 24(4.17)$ \\
\hline Plied tube & $1 / 23(4.35)$ \\
\hline Bezoar & $1 / 23(4.35)$ \\
\hline
\end{tabular}

IQR - interquartile range; PEG-J - percutaneous endoscopic transgastric jejunostomy

\section{DISCUSSIONS}

This study succeeded in its aim to investigate the PEG-J use and the occurrence of procedure complications in a group of patients diagnosed with PD Hoehn and Jahr 3 and 4 stages, receiving LCIG therapy.

A study performed by Udd M et al. analyzed the clinical outcome and complications of LCIG in sixty patients with advanced PD from the Helsinki University Hospital between 2006 and 2014 [11]. The result was that most patients were satisfied with LCIG treatment and the majority of the patients had complications including: peristomal complications: buried PEG bumper (1\%); skin problems, leaking stoma $12(20 \%)$; nonspecific infection 4 (7\%); skin infection, abscess $5(8 \%)$; granulation tissue $21(35 \%)$; tube complications: tube occlusion 13 (22\%); accidental removal of inner tube 23 (38\%); dislocation of the inner tube backwards into the stomach 5 (8\%); tube break 11 (18\%); other complications: weight loss $\geq 5 \% 18$ (30\%); neurologic symptoms 3 (5\%); pump issue 3 $(5 \%)$.

Several removals of PEG-J by the patient suggest severe mental deterioration, and discontinuation of LCIG can be considered an option. The results were similar to our study, PEG-J removals and peristomal complications being the most frequent complications.

The decision for withdrawal of LCIG might be difficult sometimes, but in most cases, at this moment, there is no benefit for the health of the patient. A close collaboration between the neurology, psychiatry, and gastroenterology department is mandatory to overcome difficult decisions like this. A study performed by Fernandez $\mathrm{H}$ et al. analyzed the safety and efficacy of LCIG in patients with advanced PD experiencing severe motor fluctuations defined as $\geq 3$ hours of daily "off" episodes at baseline written in the PD symptom diary, despite optimized treatment with available PD medications [12]. The intrajejunal tube was well tolerated and only $7.6 \%$ of the patients withdrawn from the study because of adverse effects [12].

Unfortunately, $92 \%$ of the patients reported more than one adverse effect, most commonly associated with PEG-J insertion, and decreased substantially after the first-week post-PEG-J tube 
placement, a result similar to ours. Device complications were most common in the first week after PEG-J placement and included: complication of device insertion $34.9 \%$, abdominal pain $31.2 \%$, procedural pain $20.7 \%$, nausea $16.7 \%$, excessive granulation tissue $16.0 \%$, postoperative wound infection $15.4 \%$, constipation $14.5 \%$, site incision erythema $13.0 \%$, complication of device insertion $6.5 \%$, abdominal pain $3.1 \%$, peritonitis $2.8 \%$, polyneuropathy $2.8 \%$, pneumoperitoneum $2.5 \%$ and device dislocation $1.5 \%$ [12].

A study performed by Saddi MV et al. compared two different methods of continuous intrajejunal delivery of levodopa/carbidopa. The first method, percutaneous endoscopic gastrojejunostomy (PEG-J) and the second, radiologically inserted gastrojejunostomy (RIG) are safe and efficient procedures for gastrojejunal tube placement [13]. Because the incidence of adverse events and complications were similar in both groups, the conclusion was that RIG technique is as safe and effective as PEG-J, and it can be used for patients who require LCIG when the endoscopic procedure is not recommended, or the patient does not tolerate PEG-J [13].

A study performed by Murata $\mathrm{M}$ et al. on 28 Japanese, Korean and Taiwanese patients with advanced Parkinson's disease showed that all patients had at least one adverse event and three patients discontinued LCIG therapy due to an adverse event [14]. The most frequent adverse events and complications included: incision site pain $50.0 \%$, nasopharyngitis $35.7 \%$, constipation $32.1 \%$, diarrhea $28.6 \%$, incision site erythema $25.0 \%$, weight decreased $25 \%$, dyskinesia $17.9 \%$, procedural pain $17.9 \%$, nausea and vomiting $17.9 \%$ and vitamin b6 deficiency $17.9 \%$ [14]. Our study similarly found that PEG-J complications arose in half of the patients included in our study.

A previous study that analyzed 14 patients under LCIG treatment showed that PEG-J related complications were observed in 10 patients $(71.4 \%)$. The most frequently reported complications were: J-tube related complications such as kinking (3 cases, 21.4\%), pump malfunctions ( 3 cases, $21.4 \%$ ), skin troubles in the gastrostomy ( 7 cases, $50.0 \%$ ), duodenal perforation, peritonitis, and ulcers ( 2 cases, $14.3 \%$ ) [15].

A study performed in Helsinki University Hospital between 2006 and 2014 analyzed the clinical outcomes and the complications in patients sixty with advanced PD under LCIG. 60 patients underwent a total of 156 PEG-J procedures, and 48 patients $(80 \%)$ had a total of 143 complications including: accidental removal of the J-tube in 23 patients (38\%) most common complication; $\geq 5 \%$ weight loss in 18 patients (30\%); peristomal complications: buried PEG bumper 1(1\%); skin problems, leaking stoma $12(20 \%)$; nonspecific infection 4(7\%); skin infection, abscess 5 (8\%); granulation tissue 21(35\%); tube complications: tube occlusion $13(22 \%)$; accidental removal of inner tube 23 $(38 \%)$; dislocation of the inner tube backwards into the stomach 5 (8\%); tube break $11(18 \%)$ [16]. A study performed by Fernandez HH et al. on 272 PD patients, analyzed the LCIG procedure/device-related AEs, reported for $68.5 \%$ of patients and included: primarily complication of device insertion (33.6\%), abdominal pain (26.5\%), procedural pain (20.4\%), excessive granulation tissue (15.4\%), postoperative wound infection (15.1\%), incision-site erythema $(12.7 \%)$, procedural-site reaction $(9.3 \%)$, postprocedural discharge $(7.7 \%)$, incision-site pain $(6.2 \%)$, and pneumoperitoneum $(5.9 \%)$ [17]. Our study similarly found that local erythema and allergic reaction were present.

A prospective study performed by Pålhagen SE et al. on 77 patients enrolled in a prospective, open-label study in routine medical care analyzed LCIG treatment in patients with advanced PD having as outcome that long-term safety was consistent with the established LCIG profile, although adverse effects were common and related to the device, procedure, levodopa, or laboratory evaluations [10]. The most frequent adverse event occurring in $>10 \%$ of any cohort of the study included: medical device change (41.7\%), device dislocation (27.8\%), constipation (22.2\%), anaemia (19.4\%), device-related infection (19.4\%), excessive granulation tissue $(16.7 \%)$, device occlusion (13.9\%), medical device complication (2.8\%) and medical device removal (2.8\%) [18]. Similar to our study, tube dislocation, erythema, and inflammation at the level of stoma were present.

A limitation of the study is its sample size. The results are generalizable to patients in regional tertiary centers (since the subjects in this study were collected from this setting). The small number of exclusion criteria helps for the generalizability. 
Having taken into account the clinical results of the research and also the similar findings of other studies, this study enhances the present knowledge with more evidence supporting the important frequency of complications due mainly to replacement of PEG-J, also frequent interruptions, primarily due to patient or relative refuse.

\section{CONCLUSIONS}

The most frequent complication of LCIG delivered by PEG-J was erythema and inflammation at

\section{REFERENCES}

1. Kowal SL, Dall TM, Chakrabarti R et al. The current and projected economic burden of Parkinson's disease in the United States. Mov Disord. 2013;28:311-8.

2. Karbozova K. The study of the prevalence of Parkinson disease. Parkinsonism and Related Disorders. 2016;22.

3. Rocca W. A. The burden of Parkinson's disease: a worldwide perspective. The Lancet Neurology. 2018;17(11):928-929.

4. Olanow CW, Kieburtz K, Odin P et al.; LCIG Horizon Study Group. Continuous intrajejunal infusion of levodopa-carbidopa intestinal gel for patients with advanced Parkinson's disease: A randomised, controlled, double-blind, double-dummy study. Lancet Neurol. 2014;13(2):141-9.

5. Nyholm D, Odin P, Johansson A et al. Pharmacokinetics of levodopa, carbidopa, and 3-O-methyldopa following 16-hour jejunal infusion of levodopa-carbidopa intestinal gel in advanced Parkinson's disease patients. AAPS J. 2013;15(2):316-23.

6. Braak H, Tredici K, Del Rüb U et al. Staging of brain pathology related to sporadic Parkinson's disease. Neurobiol Aging. 2003:24:197-211.

7. Stewart KC, Fernandez HH, Okun MS et al. Distribution of motor impairment influences quality of life in Parkinson's disease. Mov Disord. 2008;23:1466-1468.

8. Lo RY, Tanner CM, Albers KB et al. Clinical features in early Parkinson disease and survival. Arch Neurol. 2009;66:1353-1358.

9. Zhang X, Guo H, Xu J et al. Dopamine receptor D1 mediates the inhibition of dopamine on the distal colonic motility. Trans/ Res. 2012;159:407-14.

10. Hagell $P$, Nygren $C$. The 39 item Parkinson's disease questionnaire (PDQ-39) revisited: Implications for evidence based medicine. J Neurol Neurosurg Psychiatry. 2007;78:1191-1198. the level of stoma. LCIG therapy was interrupted primarily due to the patient or relative refuse. The most frequent reason for PEG-J replacement was tube dislocation, and rarely the plied tube, the excess of adipose tissue, or bezoar. A third of the patients received PEG-J therapy during the night, after a median time of three years, after PEG-J initiation.

\section{Acknowledgement}

All authors equally contributed to this manuscript.

Conflict of interest: none declared Financial support: none declared

11. Udd M, Lyytinen J, Eerola-Rautio J et al. Problems related to levodopa-carbidopa intestinal gel treatment in advanced Parkinson's disease. Brain Behav. 2017;7(7):e00737.

12. Fernandez HH, Standaert DG, Hauser RA et al. Levodopa-carbidopa intestinal gel in advanced Parkinson's disease: Final 12-month, openlabel results. Mov Disord. 2015;30(4):500-9.

13. Saddi MV, Sarchioto M, Serra G et al. Percutaneous Endoscopic Transgastric Jejunostomy (PEG-J) Tube Placement for LevodopaCarbidopa Intrajejunal Gel Therapy in the Interventional Radiology Suite: A Long-term Follow-up. Mov Disord Clin Pract. 2018; 5(2):191-194.

14. Murata M, Mihara M, Hasegawa K et al. Safety and efficacy of levodopa-carbidopa intestinal gel: Results from an open-label extension study in Japanese, Korean and Taiwanese patients with advanced Parkinson's disease. Ther Adv Neurol Disord. 2018;11:1756286418759315.

15. Shimamura M, Shinzaki S, Ikenaka K et al. Analysis of PEG-J associated complications in 14 adult patients treated with levodopacarbidopa intestinal gel. Rinsho Shinkeigaku. 2019;59(3):153-156.

16. Udd M, Lyytinen J, Eerola-Rautio J et al. Problems related to levodopa-carbidopa intestinal gel treatment in advanced Parkinson's disease. Brain Behav. 2017;7(7):e00737.

17. Fernandez HH, Standaert DG, Hauser RA et al. Levodopa-carbidopa intestinal gel in advanced Parkinson's disease: Final 12-month, openlabel results. Mov Disord. 2015;30(4):500-9.

18. Pålhagen $S E$, Sydow $O$, Johansson $A$ et al. Levodopa-carbidopa intestinal gel (LCIG) treatment in routine care of patients with advanced Parkinson's disease: An open-label prospective observational study of effectiveness, tolerability and healthcare costs. Parkinsonism Relat Disord. 2016;29:17-23. 